\title{
Peculiarities of membrane bioreactors' maintenance
}

\author{
Nikolay Makisha ${ }^{1, *}$ and Artem Kulakov ${ }^{2}$ \\ ${ }^{1}$ Moscow State University of Civil Engineering, Research centre "Water supply and wastewater \\ treatment”, 26, Yaroslavskoye shosse, Moscow, Russia, 129337 \\ ${ }^{2}$ Vologda State University, Department of water supply and wastewater treatment,15, Lenina str., \\ Vologda, Russia, 160000
}

\begin{abstract}
The article reveals various factors, which determine the maintenance of membrane bioreactors for treatment of domestic and industrial sewage. Contamination of membranes is a consequence of poor pretreatment, when membrane accumulates hair, scraps of rags and other fibrous materials on its' fibers and in the cavities of membrane blocks. Aeration conditions have a significant impact on the performance of the membranes, also in sufficient sludge liquor mixing leads to membrane clogging. Size of pores, material of membrane and washing peculiarities are the factors that can affect MBR operation. The costs for installation and operation of membrane modules is still the limiting factor of their wide application, however now the situation is changing. There are key differences between membrane and conventional treatment of wastewater revealed such as costs, required area and quality of treatment.
\end{abstract}

\section{Introduction}

Restoration of permeability of membranes within MBR operation is made by means of solutions of reagents, generally, oxidizing agents - so-called chemical washing. The main agents in use are sodium hypochlorite concentration of $0.2-1 \%$ or citric acid $(0.2-0.3 \%)$; caustic sodium, hydrochloric acid, various detergents and complexing agents are used rarely. The frequency of this procedure is approximately once per several months. Preventive treatment with sodium hypochlorite can be carried out more regularly - several times per month. The pressure modules are washed by circulation of the reagent solution supplied by the pump from a special tank; submersible modules are either moved to a special container or washed in place. The duration of the procedure is several hours [1]. In some cases, there is a need to take out membrane blocks and wash them mechanically to remove accumulated sediments (Figure 1).

If we focus on the main difficulties encountered in the operation of membrane bioreactors and exclude the features of the biological purification, it is possible to distinguish the following characteristic problems according to their priority:

- fouling of membranes and filters;

${ }^{*}$ Corresponding author: nmakisha@gmail.com 
- damage to the membranes or their severe pollution;

- failures of communication lines of automation systems;

- membrane blowing system failures;

- failure of blowers and aerators;

- contamination of the grids or gratings;

- recycling failure;

- failure of auxiliary membrane equipment.

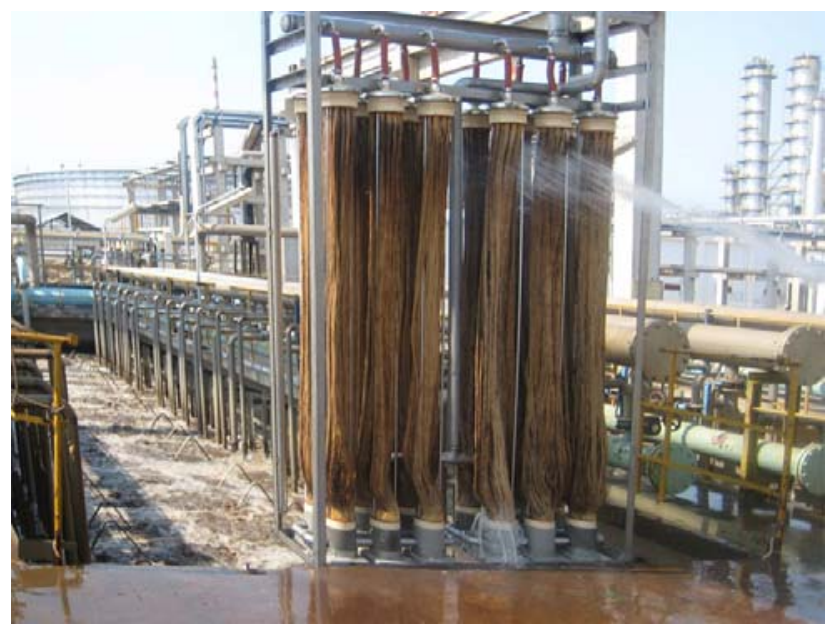

Fig. 1. Washing of membranes.

\section{Technological and economic aspects of MBR application}

Contamination of membranes and mesh filters is a consequence of poor pretreatment, when membrane accumulates hair, scraps of rags and other fibrous materials on its' fibers and in the cavities of membrane blocks [2].

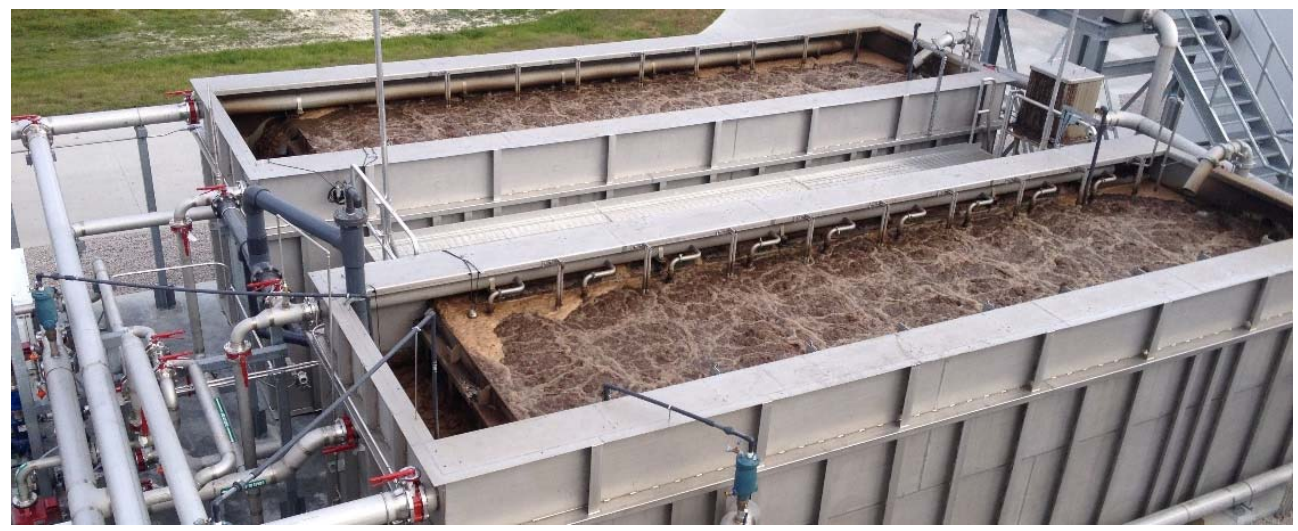

Fig. 2. Small capacity WWTP with MBR technology.

Aeration conditions have a significant impact on the performance of the membranes. Deterioration of the characteristics of the sludge mixture (for various external and internal reasons, including aeration problems) almost does not worsen the quality of the purified liquid, but leads to a deterioration in the permeability of the membranes and their clogging. 
Capital costs for the construction of a wastewater treatment plant with implementation of MBR are in the range of 600-1000 euros per $1 \mathrm{~m}^{3} /$ day depending on the capacity of the system (Figure 2). The cost of the membrane unit itself (with all accessories) is $30-60 \%$. The cost of membrane blocks is $75-150 \mathrm{Euro} / \mathrm{m}^{2}$ with an average specific capacity of $15-30 \mathrm{l} / \mathrm{h}$ per $1 \mathrm{~m}^{2}$ of membrane area [3]. The cost of domestic wastewater treatment by means of membrane modules lies in the range of $0.08-0.15$ euros per $1 \mathrm{~m}^{3}$, with smaller values obtained when using half-fiber modules (Figure 3); the total operating costs are $0.24-0.25$ euros per 1 $\mathrm{m}^{3}[4]$.

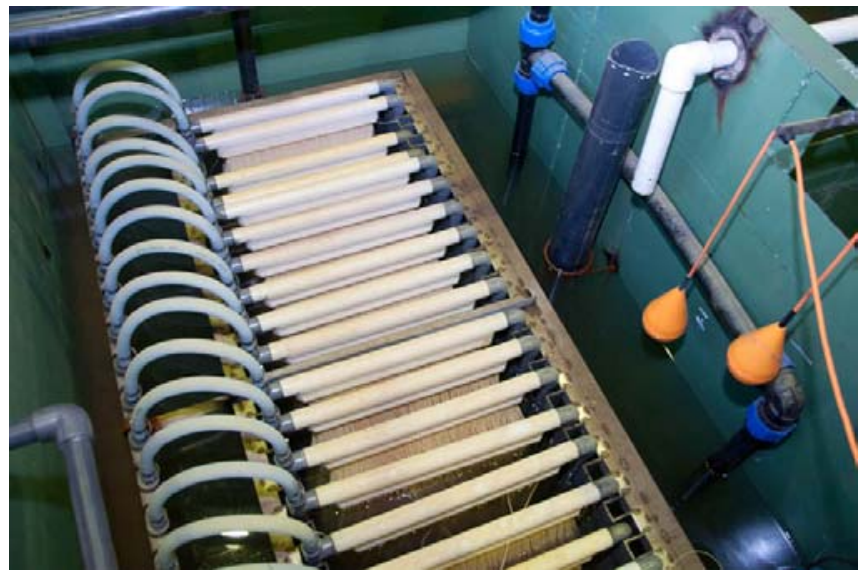

Fig. 3. Membrane inside the tank.

Membrane bioreactors are used to treat industrial wastewater, surface wastewater, industrial water of textile production, wastewater of dairies and creameries, as well as wastewater of poultry farms. Main advantages of MBR technology introduction are $[1,4,5]$ : - Improvement the efficiency and reliability of treatment facilities;

- Increase the capacity of treatment facilities by increasing the concentration of activated sludge in aeration tanks;

- Creation of compact treatment facilities, due to the replacement of secondary settling and filtration filters of various types on the membrane tertiary treatment;

- Reduction the amount of excess activated sludge.

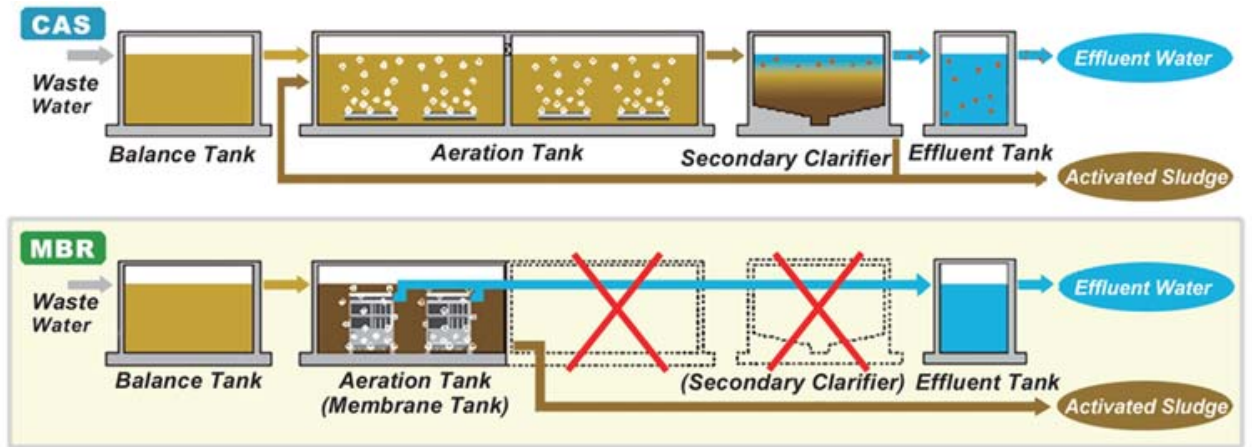

Fig. 4. Conventional scheme of treatment and MBR-scheme.

In addition to above meant advantages the following should be noted. Purified water requires additional filtration and disinfection after the conventional bioreactor. Disinfection of the treated wastewater after the classical bioreactor is made by adding sodium hypochlorite solution or by means of ultraviolet light. Sodium hypochlorite causes the need to use sorption 
filters at the end of the technology, and ultraviolet lamps may not give the necessary efficiency of disinfection. Membrane bioreactor helps in avoiding these problems with a high degree of reliability as they combine the processes of microfiltration and ultrafiltration, and a process of aerobic biological wastewater treatment [6-8].

Currently, in most industries, the costs of wastewater treatment facilities lay in the range between 5 to $30 \%$ of the cost of the main equipment and facilities. If wastewater treatment with the efficiency of $99-99.5 \%$ is needed, the cost of treatment facilities sharply increases. Capital costs and operating costs of conventional treatment facilities with a capacity of 10000 $\mathrm{m}^{3}$ /day are about 2.2 million euros, the area of these facilities is about $5000 \mathrm{~m}^{2}$ (Figure 4).

The economic effect of using MBR technology for these treatment facilities is expected to be $700-800$ thousands of euros, or about $25-30 \%$. In addition, the area needed for placement of treatment facilities will be halved.

The cost-effectiveness of MBR is driven by technology that allows:

- reducing the daily production of dry matter of active sludge and suspended solids by $22 \%$;

- using active sludge with high MLSS and SRT in the membrane bioreactor to provide less volumes of the facilities;

- total removing of bacteria and viruses (efficiency over $99.99 \%$ with ultrafiltration);

- achieving both the decomposition of organic pollutions and nitrification under the HRT of three hours due to high MLSS and SRT;

- reducing the size of the flakes of activated sludge and increasing species diversity;

- reducing of the necessary area in comparison to conventional treatment facilities;

- accepting wastewater with various initial concentrations;

- reusing of wastewater for non-drinking needs;

- eliminating secondary clarifiers, that creates conditions to develop slow-growing bacteria able to decompose complex compounds. Membrane can keep enzymes, the presence of which leads to the intensification of metabolic processes.

Application of MBR technology reduces capital costs for construction by $20-30 \%$, which gives a significant economic effect, however energy consumption grows within operation. The area of treatment facilities is reduced by $50 \%$. Thus, the efficiency of MBR is determined by its small volume facilities and the ability to vary the treatment of wastewater for different industries [7].

\section{The advanced MBR technology and application prospects}

The use of MBR for municipal wastewater utilization determines the application of new strategy to improve the quality of wastewater treatment, as well as reducing the financial costs for the construction and operation of municipal wastewater treatment facilities.

The number of municipal facilities that launched MBR operation has increased dramatically in recent years.

Wastewater treatment plants with the technology of MBR in a warm climate are less expensive with the same characteristics than in the cold. This is due to the viscosity of the liquid passing through the membrane. The minimum temperature of wastewater is the main factor in determining the number of membrane modules required. Researches on the operating modes of MBR showed that membrane fouling is one of the most difficult problems in the operation of membrane bioreactors. Operation becomes more complicated, increasing energy consumption, etc. According to the results of studies of the operation of MBR, carried out in different conditions, the intensity of membrane fouling was associated with the filtration rate of the sludge liquor [9].

Nevertheless, use of MBR technology is expanding, new types of membranes are being developed that are resistant to sediment formation and are able to operate at low transmembrane pressures, to allow using membrane methods for the treatment of low- 
contaminated wastewater.

The technology of MBR is being improved with implementation of hollow-fiber membranes grouped into cassettes (Figure 5) under which pneumatic aerators are placed. In China, a new type of membrane bioreactor has been developed and studied, in which 18 membranes are combined with a floating biofilm carrier in the form of a microfilter. High organic loads, high treatment efficiency, stability under irregular loads and a small area for the facilities characterize the new MBR. Treatment quality allow reusing treated water $[3,10]$.

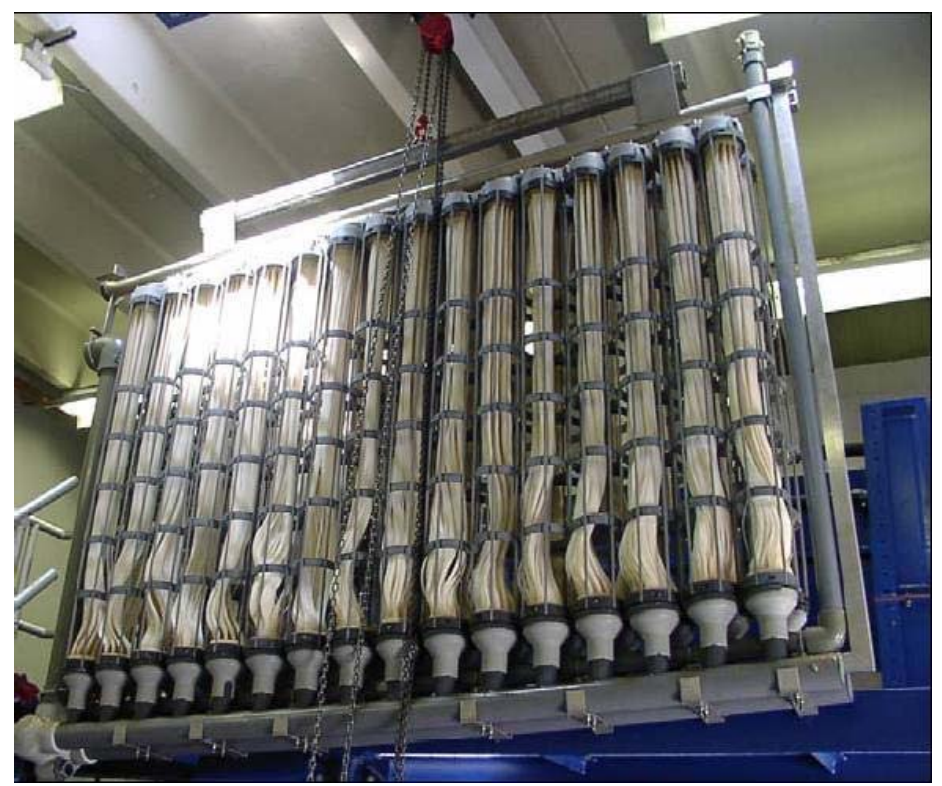

Fig. 5. Membrane cassettes.

\section{Factors affecting membrane operation}

In this section, several factors will be overviewed that have to be taken into account whenever membrane technologies are applied:

1. Material of membranes. The choice of material is strictly linked to the pollution in the incoming wastewater (in particular, the intercellular organic substances, polysaccharides and proteins) and chemical resistance to membrane modules washing. Membranes mostly have hydrophobic properties. The charge of the membrane also affects the degree of its pollution. Membrane manufacturers can also make certain alteration in the composition formula to add specific features and as a result improve their performance.

2. Size of pores of the membranes is not critical: MF membranes with a pore size of 0.1 -1 microns and UF membranes with a pore size of $0.01-0.1 \mu \mathrm{m}$ shows almost the same efficiency in removing suspended solids and microorganisms, equalized by the accumulation of sediment on the surface of the membrane in the filtration process. Reduction of pore size improves the stability of the membrane to contamination, and their hydraulic flushing can help to remove sediment from the surface.

3. Membrane permeability. The flow through the membrane is the main factor influencing the rate of formation of precipitate on its surface. There is the concept of "critical flux", above which the growth of the precipitate becomes invalid for the normal functioning of the membrane module. Many MBR facilities work with a constant performance, which is achieved by adjusting the transmembrane pressure. The pressure increase on the membrane in the process causing compression of the sediment and increase its resistance. 
4. Aeration of the membranes. The main way to control the process of membrane pollution is blowing their bubbles that remove deposits from the membrane surface and mix the surrounding fluid, improving the mass transfer. The cost of aeration / venting of the air is one of the main components of operational costs in MBR. Airflow to the membrane module is 0.2 to $1.3 \mathrm{~m}^{3} / \mathrm{h}$ per $1 \mathrm{~m}^{2}$ of membrane in it. This value depends on the amount of fluid around the membranes, the specific area of membranes, and intensity of airflow.

5. Hydraulic flushing. Flushing with a reverse flow of the filtrate is an efficient tool to avoid sedimentation taken form ultrafiltration systems for water purification. As a rule, modules with flat membranes (except roller designs) do not allow reverse flushing. The intervals between back flushing and their duration are in the range $10-60$ minutes and $15-$ 300 seconds, respectively. MBR facilities also used pulsed flushing - frequent (1 every few seconds) pulses of the reverse current of the filtrate with less than 1 second.

6. Composition of the incoming wastewater. The presence in wastewater of a large amount of easily biodegradable organic matter contributes to the formation of a larger amount of extracellular polymeric substances (polysaccharides, proteins) that can clog the ultrafiltration membrane. Since membrane detain suspended matter, and partly polysaccharides and proteins, the concentration of these substances in the bioreactor increases, which causes an increase in resistance of the produced sludge. The increase in sludge age helps to reduce contamination of the membranes by reducing the content of polysaccharides in the sludge.

\section{Conclusions}

1. Membrane bioreactors now have growing implementation worldwide that is confirmed by the advantageous features over conventional treatment like less area needed, higher efficiency of treatment and less amount of produced excessive sludge

2. A certain attention should be paid to type of membrane modules (material, size of pores, permeability) according to their characteristic features and operation peculiarities.

3. High cost and integrated control and management systems remain as limiting factors for wider MBR application.

The reported study was funded by the The Head Regional Shared Research Facilities of the Moscow State University of Civil Engineering.

\section{References}

1. A.A. Kulakov, ViST, 5, 25-30 (2013)

2. A.A. Kulakov, Wat.Ecol., 1, 61, 26-40 (2015)

3. Zaletova N.A., Zaletov S.V., Int. J. of Appl. Eng. Res., 10, 23, 43873-43874 (2015)

4. N. Makisha, D.Semenova, MATEC Web of Conferences, 144, 04016 (2018)

5. I.Gulshin, MATEC Web of Conferences, 106, 07002 (2017)

6. I.Gulshin, IOP: Earth and Env. Sci., 90, 012198 (2017)

7. A.G. Pervov, A.P. Andrianov, DWT, 35, 1-3, 2-9 (2011)

8. E. Sherbina, N. Danilina, D. Vlasov, Int. J. of Appl. Eng. Res., 10, 22, 43131-43138 (2015)

9. N. Danilina, E3sconf, 6, 02001 (2016)

10. N. Makisha, E3sconf, 6, 01002 (2016) 